\title{
L'autotransplantation de la dent de sagesse : étude économétrique comparative avec la technique implantaire
}

\author{
Brandone S, Fauxpoint G, Esclassan R, Parayre JM, Duran D. \\ (UFR d'Odontologie, Toulouse)
}

\section{OBJECTIFS :}

Comparer en termes de coût-efficacité le traitement implantaire à l'autotransplantation dentaire des points de vue du patient, du praticien et de l'assurance maladie. Proposer une stratégie globale exploitant au mieux les avantages économiques des deux techniques.

\section{MATÉRIELS ET MÉTHODES :}

Une étude coût-efficacité a été effectuée se basant sur l'activité des cabinets dentaires libéraux de la région MidiPyrénées et du centre de soins odontologiques de Rangueil à Toulouse. Cette étude est fondée sur les montants des dépenses du praticien, du patient, et de l'assurance maladie dans le cadre de la mise en place d'un implant et d'une transplantation de la dent de sagesse. Une analyse de sensibilité a été réalisée afin d'évaluer l'effet des variations dans la fréquence des complications et la pérennité de ces traitements sur le rapport coût-efficacité.

\section{RÉSULTATS :}

Dans le cas de la pose d'un implant, le montant des dépenses du praticien et de l'ensemble patient-complémentaire en cabinet libéral (respectivement 638,30 € et 2506,60 €) est supérieur au montant de ces dépenses au centre de soins odontologiques de Rangueil à Toulouse (respectivement $576,15 €$ et $2149,10 €$ ). L'autotransplantation quant à elle engendrera les mêmes dépenses pour le patient quelle que soit la structure de soins (soit $42 €$ en cas de survie pulpaire du greffon et $82 €$ s'il y a pulpectomie). Cependant, le coût horaire du cabinet libéral (environ $60 €$ ) étant deux fois plus élevé qu'au centre de soins dentaires de Rangueil (environ $27 €$ ), l'autogreffe dentaire coutera ainsi $100 €$ à $200 €$ de plus pour le praticien libéral que pour la structure hospitalière si le transplant est maintenu vital ou non. Ceci est valable quelles que soient les hypothèses de survie et de fréquence des complications étudiées. En supposant que la gestion des échecs thérapeutiques soit gérée par la mise en place d'un implant, la technique de l'autotransplantation dentaire s'avère être la moins onéreuse pour le patient. Du point de vue du praticien, la pose d'un implant suivie d'un nouvel implant en cas d'échec est l'acte qui est économiquement le plus intéressant en tenant compte de la fréquence des échecs observés.

\section{CONCLUSION :}

La transplantation dentaire est économiquement très intéressante pour le patient malgré des chances de survie du transplant d'environ 10 ans contre 15 ans au minimum pour l'implant. Le patient a pourtant tout intérêt de choisir la greffe dentaire en première intention thérapeutique car cela lui coûtera moins cher par année de survie du greffon comparé au coût de l'implant. Les dépenses du praticien seront elles aussi nettement moins élevées s'il effectue l'autogreffe dentaire en comparaison avec l'implantation d'une racine artificielle. En effet, quelle que soit la durée de survie de la dent transplantée, en cas de remplacement secondaire par un implant, le coût par dent / année remplacée restera globalement inférieur à celui d'un implant posé d'emblée tant pour le patient que pour le praticien. De plus, en retardant l'échéance de la pose de l'implant, ce dernier pourra être conservé en bouche plus tard dans la vie du patient. 